\title{
Der Anästhesienotfall - Was tun, wenn Hund oder Katze nicht mehr atmen?
}

\author{
René Dörfelt
}

Die gute Botschaft ist: Eine Atemdepression in der Anästhesie kann gut beherrscht werden! Doch dies geht natürlich nur mit dem richtigen Know-how. Wir zeigen, wie Sie den Notfall rechtzeitig erkennen und optimal handeln.

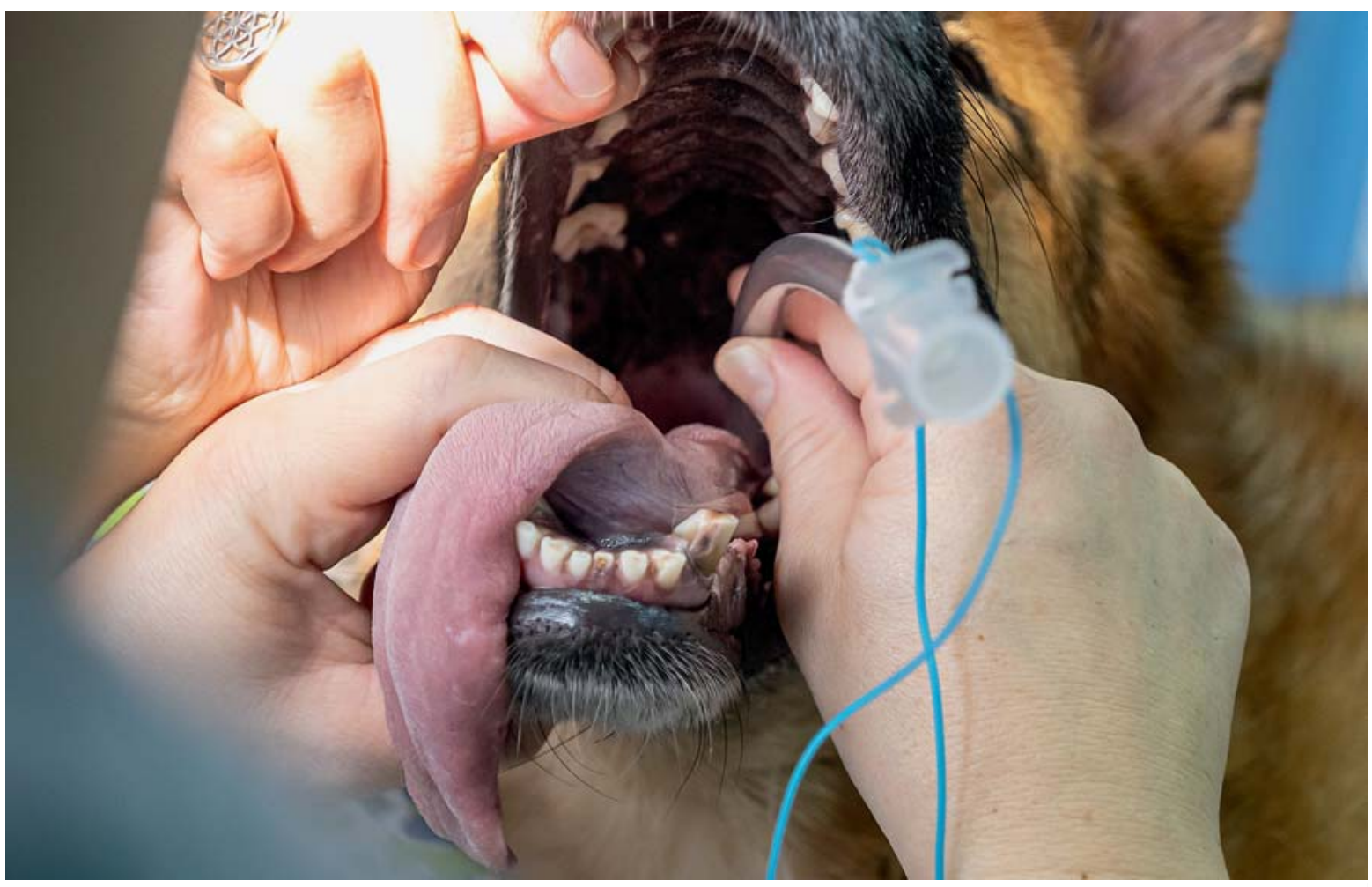

Quelle: ๑ 2019 Carlos Miguel Forero

Bei einer Anästhesie kommt es bewusst zu einer gezielten, medikamenteninduzierten „Vergiftung“ des zentralen Nervensystems. Diese hat nicht nur Auswirkungen auf das Bewusstsein, sondern vermindert auch die Funktionen und Reservekapazitäten des Herz-Kreislauf- und Atem-Systems und birgt deshalb gewisse Gefahren, z. B. die einer Atemdepression.

\section{Ursachen}

Durch eine Atemdepression wird weniger Sauerstoff aufgenommen und $\mathrm{CO}_{2}$ abgeatmet, und führt bei Tieren, die keinen zusätzlichen Sauerstoff erhalten, innerhalb von 13 Minuten zur Hypoxie. Zudem bewirkt die Anreicherung von $\mathrm{CO}_{2}$ im Blut eine Ansäuerung des Körpers. Beide Faktoren können innerhalb weniger Minuten zum Herzstillstand und somit zum Tod des Tieres führen. Auf der anderen Seite führt ein Herzstillstand auch sehr schnell 
zum Aussetzen der Atmung, sodass ein Atemstillstand auch ein Zeichen eines Herzstillstandes sein kann.

Als Gründe für Atemdepression und Atemstillstand kommen z. B. folgende Auslöser in Betracht:

- Medikamente zur Sedation und deren schnelle Verabreichung,

- sedative, analgetische und anästhetische Medikamente wie zum Beispiel Alfaxalon, Fentanyl, Ketamin, Methadon oder Propofol,

- Verlegungen der Atemwege oder Kompression der Lunge, z.B. durch eine Bohrmaschine, die auf dem Brustkorb einer kleinen Katze liegt,

- Operation an der Halswirbelsäule mit Kompression des Rückenmarks,

- mangelnde Überwachung in der Aufwachphase. Besonders bei kurznasigen (brachyzephalen) Hunden kommt es oft nach Extubation zur Verlegung der oberen Atemwege. Hier sollte nicht nur die Atembewegung, sondern auch der Luftfluss an Nase oder Maul kontrolliert werden.

\section{Merke}

Zuwenig $\mathrm{O}_{2}$ und zu viel $\mathrm{CO}_{2}$ führen in 1-3 Minuten zu Hypoxie, Azidose und evtl. zum Tod.

\section{Erkennen}

Da Anzeichen oft schon wenige Minuten vor dem eigentlichen Atemstillstand zu erkennen sind, ist bei jeder Anästhesie eine regelmäßige und engmaschige Überwachung des Patienten essenziell. Wenn der Patient nicht mehr atmet, und wir bekommen dies erst mehrere Minuten nach dem Atemstillstand mit, ist es für den Patienten oft zu spät.

\section{Atembewegung und Atemfrequenz}

Die klinische Überwachung der Atembewegungen ist die einfachste Option zur Atemkontrolle, doch leider kann dadurch nicht sicher beurteilt werden, ob genügend Sauerstoff eingeatmet und genügend $\mathrm{CO}_{2}$ abgeatmet wird. Eine hohe Atemfrequenz kann bei sehr flacher Atmung genauso eine Hypoventilation hervorrufen wie eine sehr langsame Atmung. Daher sollte nicht nur auf die Frequenz, sondern auch auf die Atemtiefe geachtet werden! Bei intubierten und am Anästhesiegerät angeschlossenen Patienten kann man Atemfrequenz und Atemtiefe durch die Bewegung des Reservoirbeutels (Atembeutels) beobachten, denn er bewegt sich synchron zur Atmung des Patienten.

\section{Kapnografie und Pulsoximetrie}

Die zuverlässigste Methode, eine Atemdepression zu erkennen, ist die Kapnografie (Messung des $\mathrm{CO}_{2}$-Gehalts der Ausatemluft). Im Falle einer Verminderung des Atemminutenvolumens steigt das $\mathrm{CO}_{2}$ an, und Werte über 55$60 \mathrm{mmHg}$ sollten auch bei gesunden Tieren nicht tole- riert werden. Wenn keine Atmung vorhanden ist, wird kein $\mathrm{CO}_{2}$ abgeatmet, die Werte sinken auf 0 , und es ist keine Kapnografiekurve zu erkennen.

Das Pulsoximeter zeigt eine Hypoventilation oder einen Atemstillstand leider erst verspätet an, denn es benötigt 1-3 Minuten, bis die Sauerstoffsättigung nach Atemstillstand absinkt. Und auch bei ausreichender Atmung kann bei Tieren, denen in der Anästhesie kein Sauerstoff zugeführt wird, eine Hypoxie, also eine Sauerstoffsättigung unter $90 \%$ vorliegen.

\section{Puls palpieren und Herz abhören}

Wichtig ist es zudem, bei Atemstillstand festzustellen, ob es zugleich auch zum Herz-Kreislauf-Stillstand gekommen ist. Hierzu sollte man sich nicht nur auf die Apparate wie EKG und Pulsoximeter verlassen, sondern den Puls manuell palpieren und das Herz auskultieren. Wenn trotz korrekter Kontakte der Elektroden und Sensoren kein EKG oder Pulsoximeter-Signal vorhanden ist und die Atmung aussetzt, kann es sich auch um einen Herz-/Atemstillstand handeln und die Wiederbelebung sollte begonnen werden.

Merke

Bei der Kreislauf-Überwachung nicht nur auf Geräte setzen: Auch Puls palpieren und Herz abhören!

\section{Behandeln}

Die einzige effektive Möglichkeit, eine Hypoventilation zu therapieren, ist die Beatmung, wobei parallel die Ursache der Atemdepression behandelt werden muss. Eine Beatmung ist nötig, wenn dem Patient mehr als 20 Sekunden lang nicht ausreichend Sauerstoff zugeführt wird.

Mit entsprechender Überwachung kann der Beatmungszeitpunkt noch effektiver eingeschätzt werden: Spätestens, wenn das endexspiratorische $\mathrm{CO}_{2}\left(\mathrm{etCO}_{2}\right)$ über $45 \mathrm{mmHg}(6 \%)$ liegt, ist eine Beatmung nötig. Genauso stellt eine Sauerstoffsättigung von unter $90 \%$ eine Beatmungsindikation dar. Sind Sauerstoffsättigung und et $\mathrm{CO}_{2}$ im Normalbereich, kann eine verminderte Atmung auch über 30-60 Sekunden toleriert werden. Alle 2060 Sekunden sollte dennoch ein Atemzug gegeben werden, damit der Kapnograph das etCO $\mathrm{C}_{2}$ bestimmen kann.

\section{Intubation}

Spätestens bei Atemproblemen und Atemstillstand sollte der Patient intubiert werden, das bedeutet, dass ein funktionstüchtiges Laryngoskop, Tuben in verschiedenen Größen bereitgelegt, und Hilfs- bzw. Haltepersonal vorhanden sein müssen. Bei Katzen oder Hunden mit schwer zugänglichen Atemwegen, hat sich die Verwendung von Stiletts oder Harnkathetern für Rüden als Führungsdraht zur Intubation bewährt. Der Tubus sollte nach Intubation mit einem Band oder einer Infusionsleitung gut an Ober- 
kiefer, Unterkiefer oder hinter den Ohren fixiert werden. Die Fixation mit Klebeband ist weniger geeignet, da sich diese im Notfall nur schlecht lösen lässt.

Um auch in Extremsituationen, wie intraoperativem Atemstillstand, zuverlässig intubieren zu können, empfiehlt es sich, die Intubation bei „normalen“ Patienten regelmäßig zu üben. Die Anästhesieleitlinien empfehlen zudem die Intubation dringend bei Eingriffen über 510 min und bei Tieren mit Atemwegsproblemen.

Cave

Nach den DVG-VAINS Leitlinien zur Anästhesie beim Kleintier sollte in jeder Praxis, die Anästhesien durchführt, die Möglichkeit zur Intubation und zur Beatmung mit Sauerstoff gegeben sein.

\section{Larynxmaske}

Eine Alternative zur Intubation stellt die Larynxmaske dar, die ohne Laryngoskop, d.h. blind eingeführt wird, auf dem Larynx liegt und mit der auch mit geringem Druck beatmet werden kann ( $\triangleright$ Abb. 1). Die Larynxmaske sichert die Atemwege nicht gegen Aspiration ab. Damit ist sie nicht zur Reanimation und bei Tieren mit Erbrechen, Megaösophagus, bei Zahnsanierungen oder Rhinoskopien geeignet. Zudem besteht beim Einsatz der Larynxmasken ein hohes Risiko des Verrutschens, sodass der Sitz permanent anhand des Luftflusses bzw. der Kapnografie kontrolliert werden sollte.

\section{Beatmung mit AMBU-Bag}

Mit der Intubation ist einen effektive Beatmung möglich, wobei die sauersoffarme Mund-zu-Tubus Beatmung weniger geeignet ist - besser ist die Gabe von Luftsauerstoff unter Verwendung eines AMBU-Bags. Zudem haben die meisten AMBU-Bags einen Anschluss für Sauerstoff (aus der Flasche) an der Rückseite ( $\triangleright$ Abb. 2 und $\triangleright$ Abb. 3). Ist der AMBU-Bag mit einem Reservoir-Beutel versehen, kann der inspiratorische Sauerstoffgehalt noch weiter gesteigert werden. Zur Beatmung sollte der AMBU-Bag nicht voll durchgedrückt werden - die meisten AMBUBags haben zwar ein Sicherheitsventil, doch dieses ist oft auf 40 bzw. $60 \mathrm{~cm} \mathrm{H} \mathrm{H}_{2} \mathrm{O}$ eingestellt. Diese hohen Beatmungsdrücke können zu deutlichen Lungenschäden führen. Einige AMBU-Bags ermöglichen eine Einstellung auf einen Maximaldruck von $20 \mathrm{~cm} \mathrm{H}_{2} \mathrm{O}$, und mit diesem Druck ist eine Beatmung ohne größere Probleme möglich. Bei der Beatmung sollte auf den Thorax geachtet werden, d. h. wenn dieser gut gefüllt erscheint, sollte die Inspiration gestoppt werden. Die Beatmungsfrequenz sollte bei 10-12 Atemzügen pro Minute liegen. Falls ein Kapnograph vorhanden ist, kann die Atemfrequenz an die Höhe des endexspiratorischen $\mathrm{CO}_{2}$ angepasst werden.

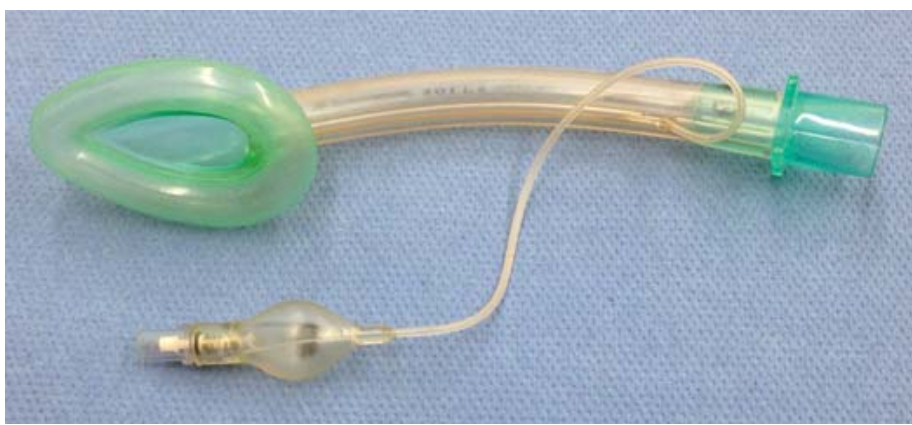

- Abb. 1 Larynxmaske zur Beatmung nicht-intubierter Patienten. Achtung: Eine Aspiration kann nicht verhindert werden und der Sitz muss laufend geprüft werden! @ R. Dörfelt

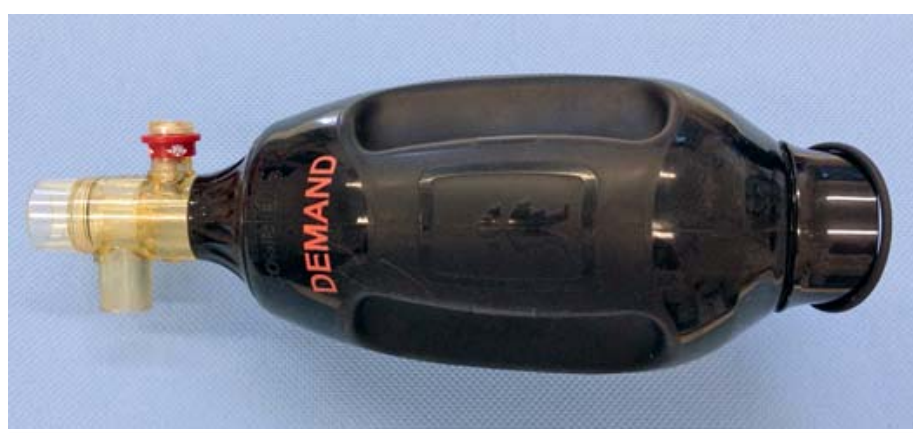

Abb. 2 AMBU-Bag mit Anschluss für Sauerstoff und Druckventil. (C) R. Dörfelt

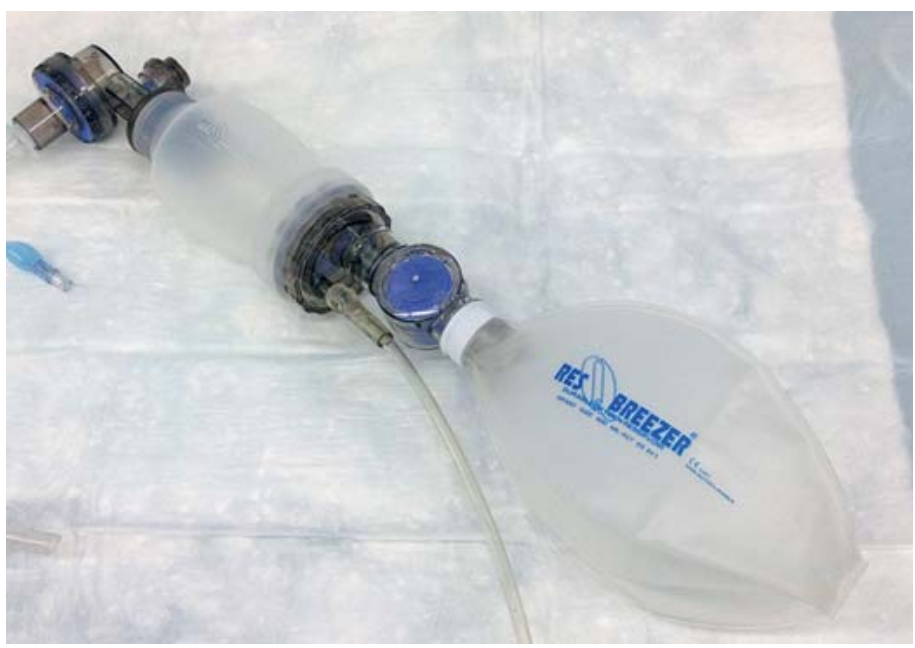

- Abb. 3 AMBU-Bag mit Anschluss für Sauerstoff, Druckventil und Reservoir-Beutel. (C) R. Dörfelt 


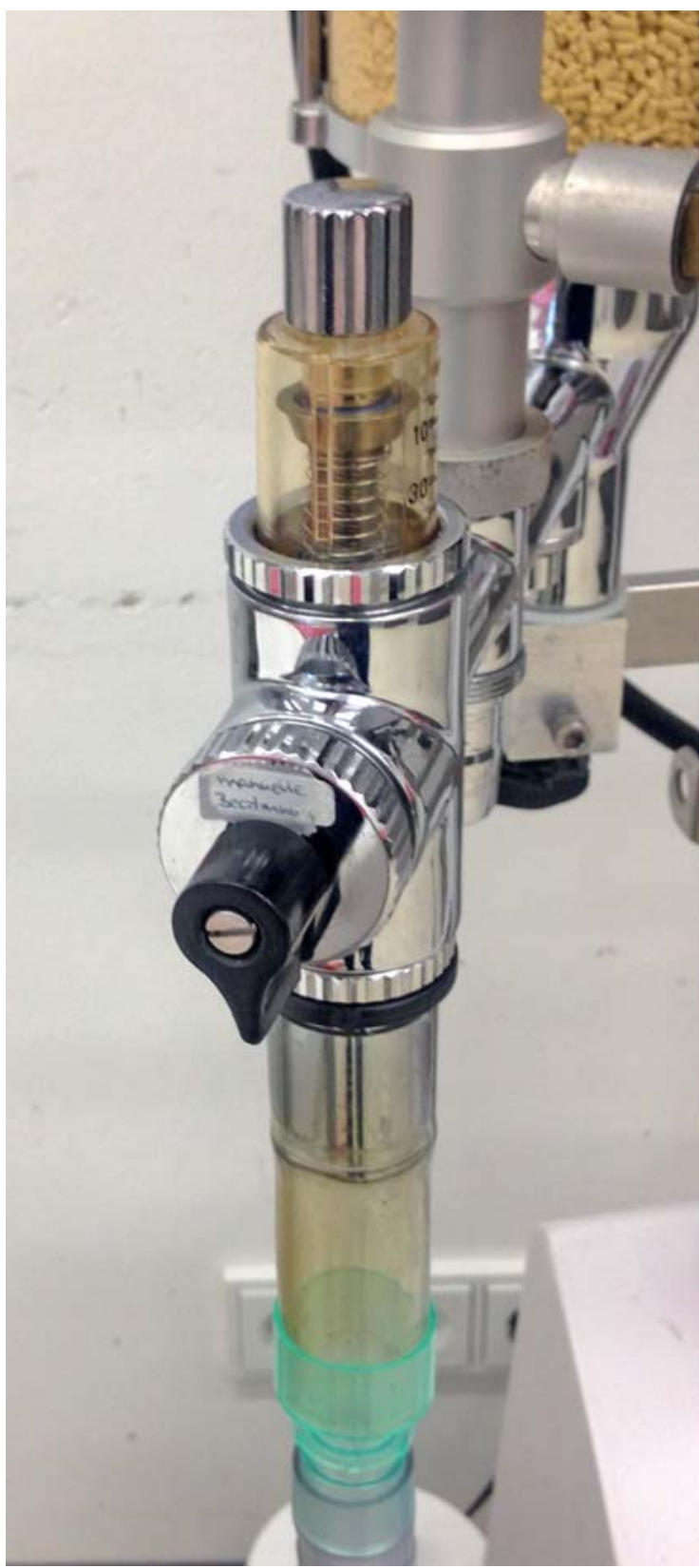

- Abb. 4 Das „POPP-Off“ oder APL-Ventil am Narkosegerät. Für die Beatmung mittels Atembeutel sollte das Ventil auf $10-12 \mathrm{~cm} \mathrm{H}_{2} \mathrm{O}$ (max. $20 \mathrm{~cm} \mathrm{H}_{2} \mathrm{O}$ ) geöffnet werden. (c) R. Dörfelt

\section{Beatmung am Anästhesiegerät}

Bei der Beatmung mit dem Anästhesiegerät wird zuerst der Sauerstofffluss erhöht, damit der Reservoirbeutel (Atembeutel) gut gefüllt ist. Nun wird das Überdruckventil, auch als „POP-Off“ oder „APL-Ventil“ bezeichnet, teilweise verschlossen ( $\triangleright$ Abb. 4 und $\triangleright$ Abb. 5). Hierbei ist

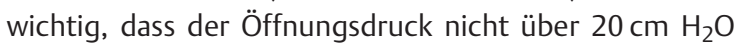
liegt, optimal sind $10-12 \mathrm{~cm} \mathrm{H_{2 }}$ O. Bei Ventilen ohne Skala muss mit jedem Atemzug der aufgebaute Druck am Manometer abgelesen und die Ventileinstellung adap- tiert werden. Der Druck auf den Atembeutel sollte so gewählt werden, dass sich der Thorax hebt. Jedoch sollte eine komplette Entleerung des Atembeutels vermieden werden, da sonst oft nicht genug Luft für den nächsten Atemzug zur Verfügung steht. Am besten eignet sich ein Atemzyklus von 2 Sekunden Einatmung und 4 Sekunden Ausatmung (Loslassen des Atembeutels). Dieser Zyklus wird wiederholt, bis alle Werte im Normalbereich sind und im besten Fall der Patient wieder selbst atmet. Je nach Situation kann dies mehrere Minuten in Anspruch nehmen.

Manche Anästhesiegeräte bieten die Möglichkeit zur apparativen Beatmung. Hier stehen je nach Gerät verschiedene kontrollierte, assistierte und unterstützende Beatmungsmodi zur Verfügung. Zudem können bei der apparativen Beatmung am Gerät die zu Patient und Eingriff passenden Parameter eingestellt werden. Anbei werden Standardeinstellungen für die apparative Beatmung aufgeführt, mit denen die meisten Patienten effektiv zu beatmen sind (nicht alle Einstellungen sind an jedem Ventilator vorhanden; Tidalvolumen, Druck und Frequenz müssen individuell an das et $\mathrm{CO}_{2}$ angepasst werden):

- Tidalvolumen: 10-15 ml/kg/Atemzug

- Atemfrequenz: 10-12/min

- Minutenvolumen: 100-150 ml $/ \mathrm{kg} / \mathrm{min}$

- Inspirations-Exspirations-Verhältnis (I-E-Ratio): 1:2 bis $1: 3$

- inspiratorischer Sauerstoffgehalt $\left(\mathrm{FiO}_{2}\right): 30-50 \%$

- Inspirationsdruck (PIP): $10-12 \mathrm{~cm} \mathrm{H} \mathrm{H}_{2} \mathrm{O}$, max. $20 \mathrm{~cm}$ $\mathrm{H}_{2} \mathrm{O}$

- positiv endexspiratorischer Druck (PEEP): $3-5 \mathrm{~cm} \mathrm{H}_{2} \mathrm{O}$

\section{Ursachen beheben}

Falls ein Medikamentenbolus der Grund der Atemdepression war, gilt es, einige Minuten beatmen, bis die Wirkungsspitze des Medikamentes abgeflacht ist. Bei zu tiefer Anästhesie sollte versucht werden, die Anästhesietiefe möglichst zu senken. Natürlich müssen auch alle anderen erkennbaren Ursachen der Atemdepression gezielt behandelt werden.

Der Einsatz von Doxapram zur Atemstimulation wird als nicht sinnvoll angesehen: Zum einen hält der Effekt nur wenige Minuten an, zum anderen wird der Sauerstoffverbrauch an Herzmuskel und im Gehirn durch Doxapram deutlich erhöht. Dies kann bei vermindertem Sauerstofftransport verheerende Wirkung für Gehirn und Herzmuskelzellen haben.

\section{Entwöhnung von der Beatmung}

Um den Patienten von der Beatmung wieder in Spontanatmung zu bekommen, ist es nötig, die körpereigene Atemstimulation wiederherzustellen. Das stärkste Atemstimulanz im Körper ist das $\mathrm{CO}_{2}$. Durch die Beatmung wird $\mathrm{CO}_{2}$ abgeatmet und somit gesenkt. Die Sauerstoff- 
sättigung trägt erst ab einem Bereich um 60-70\% zur Atemstimulation bei.

Daher ist es das Ziel, bei der Entwöhnung von der Beatmung neben der Reduktion der Anästhesietiefe, auch das $\mathrm{CO}_{2}$ in kontrollierten Bereichen (bei gesunden Tieren bis max. $60 \mathrm{mmHG}$ ) ansteigen zu lassen. Dazu werden die Atemfrequenz und die Atemtiefe vermindert, wobei Atemfrequenzen von 2-4/min meist ausreichen, um die Sauerstoffversorgung zu gewährleisten. Im Optimalfall wird der Prozess der Entwöhnung von der Beatmung mit einem Pulsoximeter und einem Kapnograph überwacht. Die Sauerstoffsättigung sollte dabei nicht unter $90 \%$ fallen.

Wenn die Tiere Sauerstoff bekommen haben, ist es ist auch möglich, sie für 30-60 Sekunden nicht atmen zu lassen, um das $\mathrm{CO}_{2}$ zu steigern. Danach wird gelegentlich ein Atemzug gegeben, um zu sehen, wo das $\mathrm{CO}_{2}$ gerade ist. Atemfrequenz und Tiefe werden so regelmäßig dem $\mathrm{CO}_{2}$ angepasst. Die gelegentlich eingebrachten Atemzüge bewirken auch eine Auslösung des Hering-BreuerReflexes, der dafür sorgt, dass nach einem Atemzug und Beatmung ein zweiter reflektorischer Atemzug erfolgt. Auch eine Kompression des Thorax erzeugt eine Reizung der Dehnungsrezeptoren in der Lunge und damit eine kurzfristige Stimulation der Atmung.

Sobald der Patient zuverlässig spontan atmet, sollte kontrolliert werden, ob er auch sein $\mathrm{CO}_{2}$ gut abatmen und die Sauerstoffsättigung unter Luftsauerstoff oder zumindest geringer Sauerstoffkonzentration halten kann. Funktioniert dies alles, kann das Tier in die Aufwachphase verbracht, oder auch die Anästhesie weiter durchgeführt werden.

\section{Fazit}

Eine Anästhesie birgt immer die Gefahr einer Atemdepression bis hin zum Atemstillstand mit Herz-KreislaufVersagen. Doch mit Know-how, passenden Geräten und optimaler Überwachung gelingt es, eine sich anbahnende Komplikation früh zu erkennen und passend entgegenzusteuern. Optimal ist es, wenn Patienten in der Anästhesie intubiert sind - das macht lebensrettende Maßnahmen schnell und effektiv möglich.

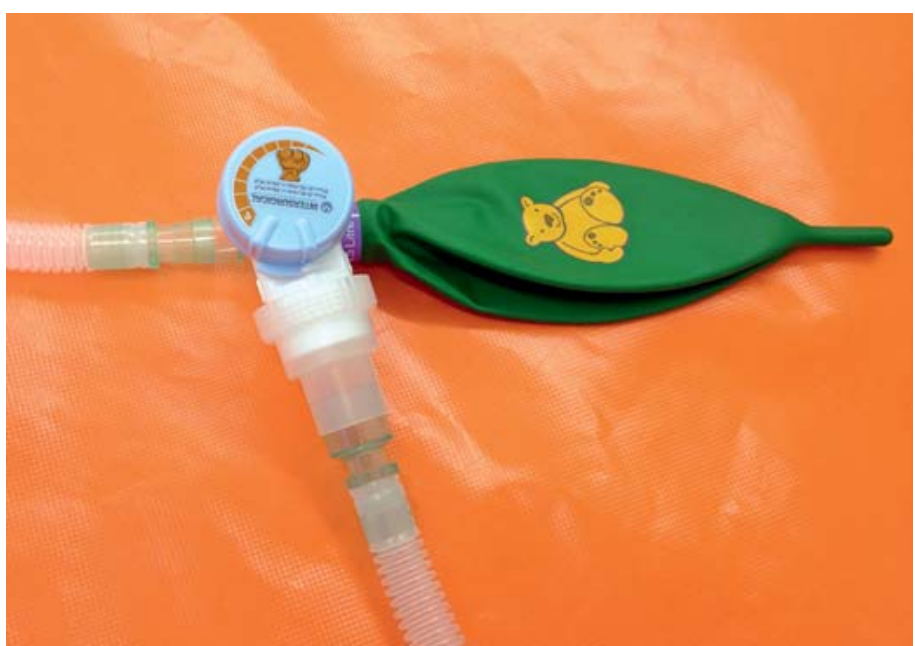

Abb. 5 Atembeutel für kleine Tiere mit geringem Volumen und einstellbarem Druckventil. @ R. Dörfelt

Korrespondenzadresse

Dr. med. vet. René Dörfelt

Dipl. ECVECC (Emergency and Critical Care), Dipl. ECVAA

(Anasthesia and Analgesia)

Fachtierarzt für Kleintiere, Fachtierarzt und Anästhesiologie, Intensivmedizin und Schmerztherapie

Ludwigs-Maximilians-Universität München

Veterinärstr. 13

80539 München

\section{Bibliografie}

DOI https://doi.org/10.1055/a-1012-3454

Team konkret 2019; 15: 13-18

(c) Georg Thieme Verlag KG Stuttgart · New York ISSN 1869-3202 


\section{Lernquiz}

\section{Der Anästhesienotfall - Was tun, wenn Hund oder Katze nicht mehr atmen?}

Testen und vertiefen Sie mit folgenden Fragen Ihr Wissen - es können mehrere Antworten pro Frage richtig sein!

Das Lösungswort steht auf Seite 28.

\section{Frage 1}

Was sind häufige Ursachen einer Atemdepression (Beispiele)?

K Mechanische Kompression des Thorax

L Medikamente

G Zu warme Zimmertemperatur

\section{Frage 2}

Was ist wichtig bei der Überwachung der Atmung? M Musik anschalten zur Beruhigung des Patienten

E Sich nicht nur auf Geräte verlassen

I Beobachtung von Atembewegung und Atemfrequenz

\section{Frage 3}

Welche Geräte helfen bei der Atem-Überwachung (Beispiele)?

$\mathrm{N}$ Kapnograph

T Stethoskop

I Pulsoximeter

\section{Frage 4}

Welche Messwerte gelten für die Einleitung einer Beatmung?

E Sauerstoffsättigung unter $90 \%$

$\mathrm{R}$ Ab einem et $\mathrm{CO}_{2}$ von $45 \mathrm{mmHg}(6 \%)$

$\mathrm{F}$ Wenn der Sauerstoffgehalt der Ausatemluft über $100 \%$ beträgt

\section{Frage 5}

Was sind Vor- und Nachteile einer Larynxmaske?

A Kann „blind“ eingesetzt werden

$\mathrm{N}$ Kann verrutschen und verhindert Aspiration nicht

W Ist eine vollwertige Alternative zur Intubation

\section{Frage 6}

Wie muss das Anästhesiegerät zur Beatmung eingestellt werden?

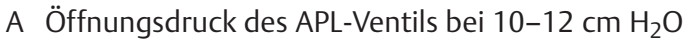

E Erhöhung des Sauerstoff-Flows

S Teilweise Schließung des APL-Ventils

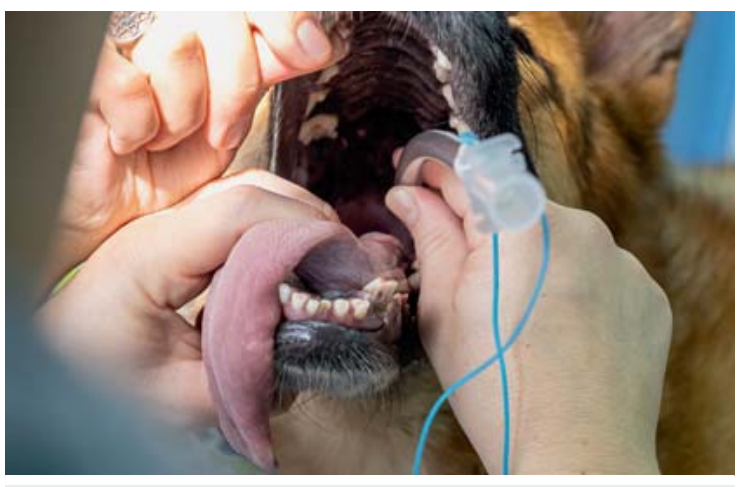

Quelle: @ 2019 Carlos Miguel Forero

\section{Frage 7}

Wie wird mit dem AMBU-Bag beatmet?

$P$ Mit einer Mischung aus $\mathrm{O}_{2}$ und $\mathrm{CO}_{2}$

$\mathrm{T}$ Anschluss an den Tubus am Patienten

H 2 sec Einatmung und $4 \mathrm{sec}$ Ausatmung

\section{Frage 8}

Wann gilt der beatmete Patient als stabil?

E Wenn alle Werte wieder im Normbereich sind

$U$ Wenn er versucht aufzustehen

S Wenn er eine normale Spontanatmung zeigt

\section{Frage 9}

Wie muss der Patient von der Beatmung entwöhnt werden?

K Entfernen von Geräten und Tubus

I $\mathrm{CO}_{2}$ bis maximal $60 \mathrm{mmHG}$ ansteigen lassen

E Kontrollierte, nachlassende Beatmung bis zur Spontanatmung

\section{Frage 10}

Was besagen die DVG-VAINS Richtlinien zum Thema Anästhesie bei Kleintieren?

I Nichts Besonderes

$\mathrm{N}$ Es sollte immer intubiert und Sauerstoff gegeben werden können

O Eine Intubation ist in den meisten Fällen unnötig 